\title{
e-Phaïstos
}

e-Phaïstos Revue d'histoire des techniques / Journal of the history of technology

IX-2 | 2021

Quel objet pour quel musée?

\section{Organiser la Méditerranée minière}

Gestion d'entreprise, contrôle des ressources et rationalisation du travail à la Société Minière et Métallurgique Peñarroya (1881-1950)

Organising the Mediterranean Mining Industry: Corporate Management, Resource Control and Work Rationalisation at the Peñarroya Mining and Metallurgical Company

\section{Francesca Sanna}

\section{(2) OpenEdition}

Journals

\section{Édition électronique}

URL : https://journals.openedition.org/ephaistos/9038

DOI : 10.4000/ephaistos.9038

ISSN : 2552-0741

Éditeur

IHMC - Institut d'histoire moderne et contemporaine (UMR 8066)

\section{Référence électronique}

Francesca Sanna, «Organiser la Méditerranée minière », e-Phaïstos [En ligne], IX-2 | 2021, mis en ligne le 26 octobre 2021, consulté le 28 octobre 2021. URL : http://journals.openedition.org/ephaistos/9038 ; DOI : https://doi.org/10.4000/ephaistos.9038

Ce document a été généré automatiquement le 28 octobre 2021.

Tous droits réservés 


\section{Organiser la Méditerranée minière}

Gestion d'entreprise, contrôle des ressources et rationalisation du travail à la Société Minière et Métallurgique Peñarroya (1881-1950)

Organising the Mediterranean Mining Industry: Corporate Management, Resource Control and Work Rationalisation at the Peñarroya Mining and Metallurgical Company

\section{Francesca Sanna}

Thèse : références bibliographiques

Francesca SANNA, Organiser la Méditerranée minière : gestion d'entreprise, contrôle des ressources et rationalisation du travail à la Société Minière et Métallurgique Peñarroya (1881-1950), Thèse de doctorat en Histoire et civilisation, Université de Paris, soutenue le 18 décembre 2020, deux volumes, un volume de texte $(650 \mathrm{p})$ et un volume d'annexes (200 p.).

\section{Directeurs de thèse}

Mathieu ARNOUX (PR, Université de Paris)

Manuela MARTINI (PR, Université de Lyon)

\section{Jury}

Judith RAINHORN, Professeure, Université Paris 1 Panthéon Sorbonne, Rapporteur Blanche SEGRESTIN, Professeure, Mines ParisTech, Rapporteur Gérard CHASTAGNARET, Professeur Émérite, Université Aix-Marseille, Examinateur Christophe GOUPIL, Professeur, Université de Paris, Président du jury

Monsieur le Président, Mesdames et Messieurs les membres du jury,

2 Permettez-moi, tout d'abord, de vous remercier pour m'avoir fait l'honneur de participer à mon jury de thèse malgré les difficultés actuelles.

3 Je suis très honorée de pouvoir présenter devant vous ma thèse de doctorat, qui porte sur le développement de l'organisation de l'entreprise contemporaine en Méditerranée, 
au prisme de l'histoire de l'une des plus grandes multinationales minières du $\mathrm{XX}^{\mathrm{e}}$ siècle : la Société Minière et Métallurgique Peñarroya. Fondée en 1881, Peñarroya (SMMP) fait partie d'une famille d'entreprises créées par la Maison Rothschild afin de diversifier ses investissements dans les secteurs minier, métallurgique et chimique dès la fin du XIX ${ }^{\mathrm{e}}$ siècle.

4 Au début, ses activités sont concentrées en Espagne, mais à partir des années 1910, Peñarroya entame une expansion de large portée en Méditerranée. Cette expansion est conçue comme un projet industriel lié à une conjoncture historique, l'entre-deuxguerres, mais reposant en même temps sur une stratégie de long terme. Face à une historiographie qui observe ces réalisations industrielles surtout au prisme des opérations financières, j'ai analysé cette expansion en partant du terrain de l'industrie. L'avancée de Peñarroya en Méditerranée implique toujours une transformation de l'exploitation absorbée, à la fois d'un point de vue technologique et gestionnaire. Ma thèse interroge ce double processus d'expansion/restructuration « au ras du sol ». À partir des objets techniques, tout particulièrement les dispositifs et les méthodes d'organisation scientifique du travail, j'ai croisé deux problématiques majeures de l'histoire contemporaine: le fonctionnement de l'entreprise et les dynamiques industrielles de l'espace méditerranéen.

5 Cette thèse est l'aboutissement d'un travail de recherche qui a débuté en septembre 2015 avec l'obtention d'un contrat doctoral, mais qui repose également sur une réflexion qui m'a accompagné tout au long de ma formation académique. Mon questionnement initial concernait le rapport entre l'espace - celui d'une terre îlotière, la Sardaigne, au centre de la Méditerranée - et des objets - les ruines d'une industrie passée, la mine. J'ai commencé à formuler ces idées comme un problème historique en Licence, puis en Master entre l'Italie et la France, à partir de l'histoire des acteurs qui avaient produit ces ruines : les entreprises minières. Ma problématique portait alors sur l'analyse des méthodes de rationalisation comme révélatrices du rapport entre l'entreprise et l'espace. Je proposai pour mon Master une étude comparée de l'application de la méthode Bedaux à la mine, en France et en Italie. Dans la comparaison, j'identifiai, comme élément fondamental dans l'explication du succès ou de l'échec des projets managériaux, l'enjeu de l'adaptabilité et de la circulation des savoirs. En même temps, les cas méditerranéens démontraient un dynamisme inédit dans la mise en place de techniques de rationalisation, constamment transformées, reformulées ou hybridées.

6 Mon projet de thèse proposait alors d'élargir l'espace d'observation et d'étudier la SMMP pour se confronter à un problème historique de grande ampleur : la question du développement industriel dans la Méditerranée contemporaine, un espace généralement considéré comme " périphérique » ou " retardataire ». La problématique de cette thèse a donc évolué en parallèle de la recherche pour se définir comme une interrogation sur l'espace méditerranéen en tant que laboratoire industriel et managérial, au prisme de l'histoire de Peñarroya.

7 J'ai ainsi imaginé une étude spatiale de l'entreprise, afin de reconstruire l'espace historique de la Méditerranée minière à partir d'une micro-analyse des objets techniques et des contextes locaux. Mais, pour faire ressortir l'architecture complexe de mon objet, j'ai adopté une approche réticulaire, à la croisée entre une perspective multi-scalaire et multipolaire. Mon approche s'inscrit dans une réflexion historiographique au sujet des circulations et des transferts, mais également dans une 
remise en perspective de la microstoria à l'échelle globale. Ma démarche a trouvé un appui très fort dans les travaux de $\mathrm{M}$. Chastagnaret, qui m'a fait l'honneur de participer à mon jury. En particulier, son analyse spatiale du comportement des entreprises minières en Espagne m'a fait, en quelques sortes, redécouvrir la géohistoire de Fernand Braudel, et il m'a suggéré un point de convergence entre les dimensions macro et micro, entre l'exploration et la prospection des objets historiques. Cet aiguillage, ou jonglage, entre les échelles constitue le cœur méthodologique de mon récit, qui trouve son unité d'analyse dans la dimension historique de l'espace.

8 Je n'ai pourtant pas défini cette approche comme un a priori de la recherche. Je l'ai développée en parallèle de l'analyse documentaire, dans le dialogue entre mes questions et les sources.

9 La recherche m'a amenée à visiter des archives dans 4 pays intéressés par les activités de la SMMP : France, Italie, Espagne et Grèce. Ces archives conservent, de manière parfois dispersée, les fonds produits par la Société et ses filiales. Les types de documents consultés sont nombreux et j'ai parfois eu l'impression de me noyer dans la prolifération des rapports techniques et administratifs, fiches, plans, correspondance, cahiers de notes, photographies...

10 J'ai pourtant essayé d'inscrire chaque document dans une logique d'analyse à trois dimensions - «locale », « supra-locale » et « réticulaire »- pour faire ressortir le macro du micro et vice-versa. De ce point de vue, par exemple, les rapports techniques des exploitations montrent l'application locale de techniques dont la forme dépend d'une circulation transnationale d'objets et de savoirs, qui ne peut être saisie que par la reconstruction d'un réseau à l'aide des rapports généraux ou de la correspondance des ingénieurs.

11 Je saisis l'occasion pour souligner l'importance des missions de recherche que j'ai pu effectuer sur les lieux de cette histoire: je n'aurais jamais pu, je le crois fortement, imaginer et comprendre l'Histoire sans observer avec mes propres yeux les territoires, et être physiquement au sein de cette géographie, en Sardaigne, en Andalousie ou en Attique.

12 Les résultats de ce travail peuvent être saisis en 4 volets :

- Les techniques d'OST au fondement du management d'entreprise ;

- La Méditerranée comme laboratoire managérial ;

- Le travail humain, objet fuyant de la rationalisation;

- Le management comme fait social et la technique comme objet négocié.

13 J'ai toujours eu un certain goût pour le démontage des objets et, d'une certaine manière, $\mathrm{j}$ 'ai fait subir aux techniques de la rationalisation le même sort: ma recherche a débuté par le démontage des techniques ayant une place centrale dans la stratégie de rationalisation de la SMMP. J'ai déconstruit les composantes - par exemple les célèbres coefficients d'effort de la méthode Bedaux - et leur histoire, en partant de la conception pour arriver aux obstacles et conflits intervenus dès leur application. Cette déconstruction m'a permis d'observer que ces techniques posent les bases pour l'architecture des systèmes managériaux, par l'enjeu de l'évaluation et de la mesure. Elles offrent, en effet, des outils ou des langages calculatoires ayant une aspiration de représentativité universelle (par exemple, par rapport à la valeur de l'effort au travail), et elles satisfont une exigence de comparabilité qui demeure à la base de l'une des fonctions fondamentales du management: la coordination. La stratégie de la SMMP inscrivait pourtant ces outils au sein d'un système qui les montraient enfin comme des 
objets en mouvement, influencés par les circulations des savoirs et par les dynamiques sociales. Dans ce cadre, les voyages et les visites des ingénieurs autour des exploitations de la Société représentent l'un des vecteurs de cette circulation. Dans la géographie dessinée par les destinations visitées, les rapports mettent en scène le mode de fonctionnement de l'entreprise dans l'espace à travers ses acteurs.

C'est ainsi que j'ai pu observer la Méditerranée comme laboratoire managérial, deuxième volet de lecture de ma thèse.

La SMMP est un "produit de la crise » de la fin du XIX ${ }^{\mathrm{e}}$ siècle et, en même temps, une création face à la crise : une solution entrepreneuriale d'expansion et rationalisation dont le but est le monopole du marché. Elle cible le contrôle des ressources minières métallurgiques, qu'elle réalise par l'organisation de circuits industriels axées sur des pôles et des flux. Les pôles sont organisés comme des microsystèmes: ils sont interdépendants, mais potentiellement autosuffisants, grâce à l'association entre services d'exploitations et d'approvisionnement. La transformation managériale de l'entreprise est, pour la SMMP, un produit autant de la crise que de l'espace méditerranéen, car les techniques sont à la fois adaptées au terrain et reformulées pour servir à construire un espace industriel. Dans cette perspective, la Méditerranée n'est plus un arrière-front en retard, mais un laboratoire managérial, où l'entreprise formule des solutions de gestion originales au lieu d'imposer des modèles prédéterminés. Cela explique, par exemple, la persistance des clivages technologiques entre exploitations et, en même temps, l'impulsion à l'innovation et à la dissémination de l'expertise managériale.

16 Tout en développant sa verticalisation métallurgique et sa diversification chimique, la SMMP demeure encore dans cette période une entreprise fortement minière, où la mécanisation s'instaure lentement. Le travail humain reste toujours le moteur de l'entreprise. Cela explique en partie le choix d'une rationalisation via l'OST, pour maitriser l'enjeu de l'efficacité de la ressource travail. Cette réflexion constitue le troisième volet de mon analyse : le travail humain, objet fuyant de la rationalisation. Le corps au travail et son dressage sont repensés par l'entreprise à l'aide des techniques d'évaluation, pour déterminer un modèle optimal du travail. Pour comprendre ces transformations, j'ai approfondi l'objet de ces techniques: le travail, l'effort et la fatigue humaine. Je l'ai fait en repositionnant mes questions sur le plan d'une histoire des sciences et d'une histoire du corps ouvrier. J'ai ainsi analysé les procédés de recodification du corps au travail que les ingénieurs de la SMMP effectuent au travers d'une mathématique de l'efficience, qui traduit les caractéristiques psychophysiologiques des travailleurs en paramètres comparables, développées à partir du langage des systèmes d'OST. Pour comprendre cette mathématique, j'ai suivi la voie de l'interdisciplinarité pour enfin découvrir que les ingénieurs utilisaient et modifiaient des lois scientifiques, comme celle de la moindre action, pour comprendre l'effort au travail. Je dois pour cela remercier M. Herbert, professeur au LIED, et M. Goupil, qui m'a fait l'honneur d'être Président de mon jury, car je n'aurais jamais pu déchiffrer mes sources sans leurs conseils.

17 Cette volonté de quantification de l'effort au travail investit également les pratiques plus traditionnelles du paternalisme, visant à intégrer l'espace privé des ouvriers parmi les facteurs qui affectent l'efficience au travail. Chez la SMMP, j'ai étudié le développement d'une sorte de paternalisme rationalisé, où chaque initiative devient un dispositif auxiliaire pour la gestion de l'énergie humaine, ainsi ramenée à l'état de 
facteur d'estimation. Face à cet apparat, le travail demeure un objet fuyant, car nul effort quantificateur n'aboutit à la disparition de la fatigue et de l'épuisement corporel, mais seulement à une relocalisation, vers l'espace domestique ou vers la sphère de responsabilité individuelle du travailleur.

Ce système intégré, qu'on pourrait définir de management total, ne se réalise pourtant pas sans confrontation sociale ; ce qui brise en partie son aspiration totalitaire. Dans ma thèse, je propose une lecture de la technique comme "négociation ", où les ouvriers apparaissent dans leurs actions de contestation, d'opposition et d'évasion envers ce système. Ce sujet fait partie du quatrième volet de lecture : le management comme fait social et la technique comme objet négociée. En particulier la communauté minière devient foyer de résistance à la fois sociale et économique, qui défie le paternalisme de la company town. J'ai en ce sens proposé une étude des pratiques matérielles et quotidiennes des ouvriers, par exemple l'agriculture domestique et la pluriactivité, comme autant de stratégies de sortie par rapport au système totalisant du management d'entreprise. L'étude des contestations dans différentes communautés met également en lumière que, en l'absence d'un levier plus politique, la négociation se joue surtout au niveau de la maîtrise et de la circulation du savoir technique. Cependant, c'est d'abord dans les micro-conflits, comme l'insubordination ou les fraudes d'enregistrement, que l'on saisit la portée de la négociation de la technique comme fait social, et comme fait quotidien. Dans cela, j'ai voulu participer à une tentative de rénovation méthodologique des études sur le monde de la mine. À la lumière de mes résultats, j'estime que l'approche micro-historique et connectée, voire micro-spatiale et réticulaire, permet de surmonter l'une des limites et des dangers qui souvent affecte l'histoire minière : l'enfermement local.

Dans cette lecture sociale, je me suis intéressée également aux ingénieurs. J'ai montré comment ils concourent à façonner les stratégies de l'entreprise à partir d'un travail de terrain, qui adapte et réinvente les techniques et les technologies. Leurs profils et leurs activités s'inscrivent pourtant dans la transformation de l'entreprise au $\mathrm{XX}^{\mathrm{e}}$ siècle : la séparation entre fonctions de contrôle et propriété, direction et exécution. À la SMMP, j'ai observé une période de transition, où le clivage entre managers et techniciens commençait juste de s'ouvrir. Les ingénieurs ressortent ainsi comme les co-animateurs de la négociation sociale qui façonne les techniques et, finalement, l'entreprise. Certaines sources m'ont permis de faire ressortir les ingénieurs non seulement comme groupe ou figure professionnelle, mais comme individus. Ces sources sont autant de terrains de chasse de l'historien, pour utiliser une métaphore célèbre, dans lesquelles l'on peut saisir les nuances de la personnalité des individus ou leur manière d'agir et de penser dans l'histoire. J'admets pourtant que mon analyse d'histoire sociale aurait pu être plus précise. Je reconnais, comme le souligne Mme Rainhorn dans son pré-rapport, que j'aurais pu mieux mettre en valeur certaines sources, comme les entretiens que j'ai utilisés dans les chapitres consacrés aux ouvriers.

20 Cette thèse est, enfin, le récit d'une exploration. Mes premiers objets de recherche ont été les techniques, les acteurs et les événements locaux comme autant d'éléments pour comprendre la rationalisation industrielle en Méditerranée. Mais c'est dans la mise en réseaux que tous ces éléments se sont articulés comme des microcosmes qui composent et reflètent leur espace d'existence, à la fois l'entreprise et la Méditerranée. 
21 Pour conclure, je voudrais proposer quelques perspectives d'ouverture, car beaucoup reste à faire : en particulier, je vois trois pistes possibles de recherche :

- En premier lieu, la piste sociale: une étude plus détaillée des communautés minières méditerranéennes correspondrait de manière symétrique à l'approche méthodologique que j'ai mobilisée dans cette thèse.

- En deuxième lieu, la piste socio-politique: l'aspect politique de la rationalisation, ou l'influence du politique dans la rationalisation, apparaissent en moindre mesure dans ma thèse par rapport à la question technique, par exemple. En même temps l'élargissement géographique de l'analyse, que j'envisage pour pénétrer les côtes méridionales de la Méditerranée, posera sans équivoque la confrontation à l'enjeu colonial. Il s'agira donc d'intégrer cette réflexion dans la complexité multi-scalaire du cadre que je viens de vous présenter.

- Enfin, je crois voir une piste environnementale : le fonctionnement des "systèmes mines » comme je les ai dénommés dans la thèse, pourrait à mon avis être relu au prisme des ressources: le bois, l'eau, le charbon. Je me propose d'approfondir cette piste si j'en ai l'occasion dans le futur.

En vous remerciant pour votre attention, je serais heureuse de répondre à vos remarques et à vos questions.

\section{RÉSUMÉS}

Entre le milieu du XIXe siècle et les années 1950, la Méditerranée participe à l'un des phénomènes les plus dynamiques de l'industrie contemporaine : le développement combiné de la mine et du rail dans l'espace européen. Dans ce contexte, on assiste, surtout à partir de 1850, à une véritable ruée vers l'exploitation minière, caractérisée par la prolifération d'entreprises de toute taille et d'acteurs issus d'horizons variés qui s'engagent dans le secteur minier. La Société minière et métallurgique Peñarroya (SMMP) est l'une de premières multinationales européennes dans le secteur des métaux et la première multinationale méditerranéenne spécialisée dans l'extraction de minerais non-ferreux. Dans sa dynamique d'expansion, la SMMP met en place des processus de restructuration et de mise en réseaux des exploitations et des compagnies absorbées ou contrôlées, qui deviennent parfois des véritables filiales. Elle construit ainsi une architecture industrielle et financière complexe et multipolaire, qui connecte les fragments et les réseaux de la Méditerranée minière du siècle précédent. Ainsi entrelacées dans les mailles de ce réseau, les interconnexions méditerranéennes se canalisent en circuits industriels, qui transforment et propulsent les flux de ressources minières vers le marché. Le réseau est donc organisé afin de garantir un contrôle des ressources et une gestion de leurs flux sur le terrain des sites miniers, dans la continuité des complexes techniques que l'entreprise élabore et met en œuvre dans l'administration de la propriété et de l'espace productif. L'un des instruments de cette stratégie est la rationalisation de la gestion de la main-d'œuvre, que la SMMP décline selon l'objectif et le contexte d'application, mais principalement dans les formes de la mécanisation et de l'organisation scientifique du travail (OST). À travers l'histoire de la SMMP, l'enquête porte donc, dans une perspective à la fois globale et micro-analytique, sur l'espace minier méditerranéen comme laboratoire d'outils managériaux. Est ainsi proposée une histoire spatiale 
de l'entreprise, où l'organisation s'affirme comme objet historique pour interroger l'espace et les transformations économiques, techniques et sociales de la Méditerranée contemporaine.

Between the mid-19th century and the 1950s, the Mediterranean mining basin participated in the combined development of mining and railways, one of the most dynamic phenomena of contemporary industry. In this context, especially from 1850 onwards, we observe a mining rush, in which companies of all sizes and players from various backgrounds were involved in mining operations. The Peñarroya Mining and Metallurgical Company (SMMP) is one of the leading European multinationals in this sector and the first Mediterranean multinational specialised in the extraction of nonferrous minerals. The SMMP implemented its structure by restructuring a network of existing companies, which sometimes become real subsidiaries. The SMMP built a complex and multipolar industrial and financial architecture, which connected the fragments of the Mediterranean mining basin. Those interconnections, converted into industrial circuits, transformed and propelled the flow of mining resources into the metallurgical verticalisation and, eventually, into the market. To this extent, management was the key to guarantee the control of the resources and the continuity of the technical processes within the administration of the productive space. Rationalisation and scientific management was used by the SMMP to develop its managerial structure. Throughout the history of the SMMP, this thesis focuses, both in a global and micro-analytical perspective, on the Mediterranean mining area as a laboratory of managerial tools. In a sort of a spatial history of the Company, scientific management asserts itself as a historical object to question the economic, technical and social transformations of the contemporary Mediterranean.

\section{INDEX}

Mots-clés : histoire des techniques, mine, industrie, rationalisation, travail, histoire des entreprises, histoire du travail, organisation scientifique du travail

Keywords : history of technology, mine, scientific management, labor science, labor history, industrial history

Thèmes : Positions de thèse

\section{AUTEUR}

\section{FRANCESCA SANNA}

Docteure en Histoire et Civilisation, Francesca Sanna est post-doctorante au laboratoire Lab'Urba à l'Université Gustave Eiffel-UPEC. Également spécialiste d'archéologie industrielle et patrimoine, ses recherches portent principalement sur l'histoire d'entreprise et du management, à l'issue d'une thèse au sujet de la Méditerranée minière contemporaine et de la Société Minière et Métallurgique Peñarroya, soutenue à l'Université de Paris en décembre 2020. Elle mène actuellement une recherche sur l'histoire des transports et de la logistique chez La Poste. 